\title{
CVaR-based stochastic wind-thermal generation coordination for Turkish electricity market
}

\author{
Aycan AYDOĞDU', Osman Bülent TÖR ${ }^{2}$, Ali Nezih GÜVEN¹
}

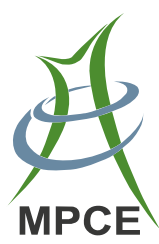

\begin{abstract}
Uncertainties in wind power forecast, day-ahead and imbalance prices for the next day possess a great deal of risk for the profit of generation companies participating in a day-ahead electricity market. Generation companies are exposed to imbalance penalties in the balancing market for unordered mismatches between associated day-ahead power schedule and real-time generation. Coordination of wind and thermal power plants alleviates the risks raised from wind uncertainties. This paper proposes a novel optimal coordination strategy by balancing wind power forecast deviations with thermal units in the Turkish dayahead electricity market. The main focus of this study is to provide an optimal trade-off between the expected profit and the risk under wind uncertainty through conditional value at risk (CVaR) methodology. Coordination problem is formulated as a two-stage mixed-integer stochastic programming problem, where scenario-based wind power approach is used to handle the stochasticity of the wind power. Dynamic programming approach is utilized to attain the commitment status of thermal units. Profitability
\end{abstract}

CrossCheck date: 29 October 2018

Received: 21 May 2018/Accepted: 29 October 2018/Published online: 27 December 2018

(C) The Author(s) 2018

$\bowtie$ Osman Bülent TÖR

osman.tor@epra.com.tr

Aycan AYDOĞDU

aycanaydogdu@gmail.com

Ali Nezih GÜVEN

guven@metu.edu.tr

1 Department of Electrical and Electronics Engineering, Middle East Technical University, Ankara 06800, Turkey

2 The Bucharest University of Economic Studies, Bucharest, Romania of the coordination with different day-ahead bidding strategies and trade-off between expected profit and CVaR are examined with comparative scenario studies.

Keywords Conditional value at risk (CVaR), Windthermal coordination, Electricity market, Wind power, Thermal power

\section{Introduction}

Participation of wind-based power plant in electricity markets offers diverse privileges for utilities and end-use costumers. However, trading of wind power in electricity markets is a challenging issue. Most wind power producers prefer trading electricity with grant-in-aid fixed feed-in tariff or long-term agreements to guarantee their profits against price fluctuations in short-term electricity markets $[1,2]$. Such risk-free long-term contracts usually have lower selling prices compared to those in short-term electricity markets. On the other hand, participation in dayahead (DA) electricity markets imposes a significant degree of risk for wind power producers. There are three main risk sources that the producers are faced with, namely, uncertainties in the forecasts of wind power, DA price, and balancing market price for the next day [3]. Contrary to the wind power generation, thermal generation is highly controllable and flexible. However, due to uncertainty and variability in market prices, thermal units are also subjected to risk of low profit or loss in DA markets [4]. Coordination facilitates thermal units to contribute to the revenue of a generation company by balancing wind generation deviations at periods of low thermal profitability. Wind generation, on the other hand, utilizes thermal units to avoid high imbalance penalties in 
balancing market with the coordination. Consequently, wind-thermal coordination has been found beneficial under wind and price uncertainties [5].

DA markets mandate participants to declare their generation schedules for the next day several hours before the start of the operational day. Time difference between the submission of bids and real-time operation ranges from 14 to 38 hours. For example, in Spanish DA market, bids are submitted a day before at 10:00 a.m. [5]. It is 11:30 a.m. for Turkish DA market case [6]. However, hourly wind power generation can be forecasted with a mean absolute error in the range of $15 \%-20 \%$ for a single plant from one day before; thus, deviations from DA schedule inevitably occur in actual generation [7]. Consequently, wind power producers are exposed to high imbalance penalties in balancing markets because of the uncertain wind forecasts. Likewise wind uncertainty, imbalance prices are also highly volatile and unpredictable. One of the reasons for this is that the amount of energy traded and the number of participants in balancing markets are relatively low compared to DA markets. Secondly, dual pricing mechanism, which Turkish balancing market has been practicing, makes imbalance prices even more difficult to estimate due to almost random nature of the direction of overall imbalances of the producers and the system.

There are various studies in literature for hedging risks associated with wind uncertainty in DA markets. Authors in $[1,3,8,9]$ suggest bidding strategies for multiple shortterm markets for wind units alone. In addition to this, coordinating wind units with different generation types are widely suggested in recent studies. In $[8,10,11]$, pumpedstorage and hydro-power units are proposed to complement the wind generation because of their capability to compensate imbalance power. There are other studies in $[5,12]$ that combine wind and thermal units to reduce imbalance penalties caused by wind deviations; however, they are not directly applicable to Turkish DA electricity market due to the different imbalance price mechanism. In addition to this, most of the previous research studied the wind-thermal coordination from an independent system operator (ISO) perspective [13, 14].

This paper contributes to the state-of-art with coordinating wind and thermal units by adapting conditional value at risk $(\mathrm{CVaR})$ concept - a mathematical approach to optimize risk of profit - to control profit variation in DA markets from the view point of generation company. For this purpose, a stochastic programming procedure considering Turkish DA market mechanism is developed. Moreover, realistic scenario studies are carried out to test the profitability of coordination and the performance of the solution algorithm under different risk measures. Results show that it is more profitable to coordinate wind and thermal units for DA and balancing markets than participation of wind and thermal units independently.

The rest of the paper is organized as follows. Electricity market mechanism in Turkey is summarized in Section 2. Section 3 presents detailed description of the problem formulation. In Section 4, the algorithm developed to solve the coordination problem is introduced. In Section 5, numerical studies are carried out and comparisons are made to investigate the benefit of coordination and the effect of risk attitude of generation company on CVaR and expected profit. Finally, concluding remarks are given in Section 6.

\section{Turkish electricity market}

In Turkish electricity market, participants can trade electricity via bilateral contracts, DA market, ancillary services market (for primary and secondary frequency control) and balancing market. The aim of the DA market is complementing bilateral agreements to ensure daily electricity supply and demand balance of the system. Balancing market manages tertiary reserves of attendants which preserve system supply/demand balance and security. Both DA and balancing markets are coordinated by the market operator and national load dispatch centre. Participation in the DA and the balancing markets is not mandatory for both supply and demand sides.

In Turkish DA power exchange market, market clearing price (MCP) is initially determined by ignoring network constraints. Market participants submit their supply and demand volumes remaining from bilateral transactions to the DA market. Participants bid power quantities (MW) and their corresponding prices $(\$ / M W h)$ that they are willing to sell or buy. At the end of the auction period, the market operator puts these quantity-price offers in order, starting from the least-cost for generator bids and highest price for buying bids. The highest demand bids are matched with the lowest supply bids in terms of price. MCP, which is equal to DA price in this case, corresponds to the point that ordered demand and supply offers are met (i.e., marginal point).

Supply and demand sides can submit both up-regulation and down-regulation bids into the market. There are certain rules in up-regulation and down-regulation bids. Price for up-regulation bid of an hour must be equal or more than the DA price of that hour. On the contrary, down-regulation price of an hour must be equal or lower than DA price of that hour. Up-regulation and down-regulation bids of qualified participants are arranged in increasing and decreasing order based on their price by market operator of Turkey, respectively. The hourly price determined by the system direction and amount of deficit is called system 
marginal price (SMP). Network constraints including secondary reserves and transmission over-loadings at steady state and significant contingencies are considered in determining the upward/downward regulation orders in Turkish electricity market, as illustrated in Fig. 1.

\section{Problem formulation and methodology}

\subsection{Two-stage stochastic programming}

To make optimal decisions in the presence of uncertainties and tackle the uncertainties in electricity markets, two-stage stochastic programming is widely used [1, 5, 11, 15-18]. First stage decisions, which are also known as "here-and-now decisions", are made before the wind generation is realized. Scenarios that represent the probability distribution of the wind power forecast are generated in the first stage for realization of plausible wind power scenarios in the second stage. The first stage decisions involve the DA power bid and the thermal unit commitment (UC). DA price, imbalance-up and imbalance-down prices, and the risk preference of generation company are the inputs to the first stage, and its objective is to maximize the expected profit.

Second stage decisions, which are also known as "waitand-see decisions", are given according to deviations in wind speed. Second stage decisions involve economic dispatch (ED) of thermal units. Results of the first stage, the DA power bid and thermal commitment decisions are deterministic inputs to the second stage. Two-stage stochastic programming model for wind-thermal coordination problem is summarized in Fig. 2.

\subsection{CVaR}

$\mathrm{CVaR}$ is frequently used to handle optimization problem in case of uncertainties. This method is a useful tool in electricity markets due to its linearity and other superior mathematical properties $[1,3,5]$. CVaR is correlated to the value at risk $(\mathrm{VaR})$ which provides information about low profits or large losses that generation company may incur. $\mathrm{VaR}$ measures the potential minimum profit for a given confidence level $\alpha$. On the other hand, CVaR, which is also known as the mean excess loss, mean shortfall, or tail VaR, is measured as the weighted average of expected profits

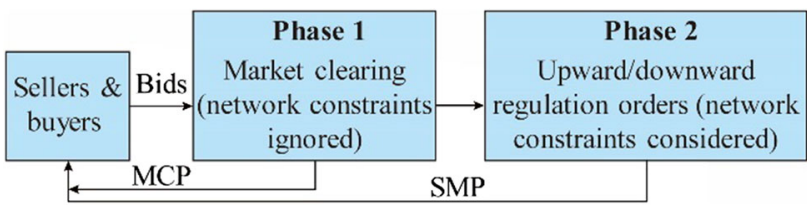

Fig. 1 Hourly-based DA Turkish electricity market

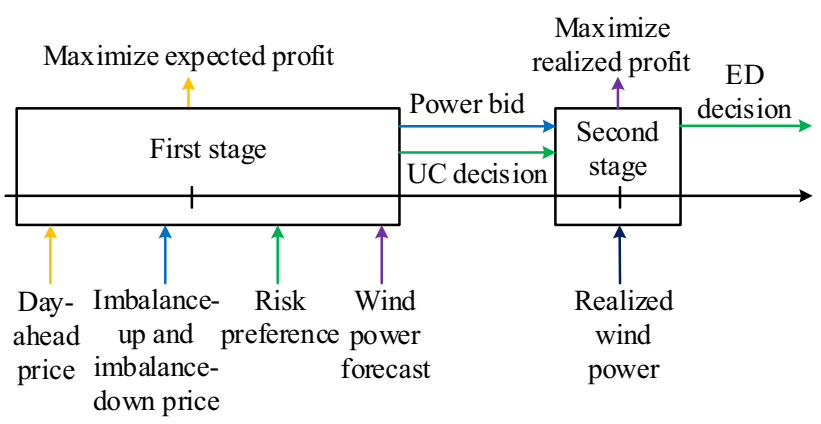

Fig. 2 Two-stage stochastic programming model for wind-thermal coordination

lower than VaR. In comparison with VaR, CVaR calculates the risk beyond $\mathrm{VaR}$ by looking at the tail of distribution. Mathematically speaking, at a confidence level $\alpha, \mathrm{CVaR}$ is the expected value of conditional profits that does not exceed VaR with a probability of $1-\alpha$. Value of $\alpha$ is proportional to the risk aversion attitude of generation company. Maximization of CVaR of profit in optimization problems is formulated as:

$\max C V a R_{\alpha}=\zeta-\frac{1}{1-\alpha} \sum_{s=1}^{N_{\mathrm{S}}} \pi_{s} \eta_{s}$

s.t.

$-P_{s}+\zeta-\eta_{s} \leq 0 \quad \forall s$

$\eta_{s} \geq 0 \quad \forall s$

where $\mathrm{CVaR}_{\alpha}$ is the $\mathrm{CVaR}$ at the $\alpha$ confidence interval; $s$ and $N_{\mathrm{S}}$ are the scenario index and the total number of scenarios, respectively; $\zeta$ is an auxiliary variable whose optimal value is equal to $\mathrm{VaR} ; \eta_{s}$ is the difference between $\mathrm{VaR}$ and $P_{s}$ which is equal to the profit of scenario $s$ with the corresponding probability of $\pi_{s}$.

$\mathrm{VaR}$ and $\mathrm{CVaR}$ parameters are illustrated in Fig. 3, where PDF and CDF stand for probability density function and cumulative density function, respectively. Assumptions made in the formulation are given explicitly as follows.

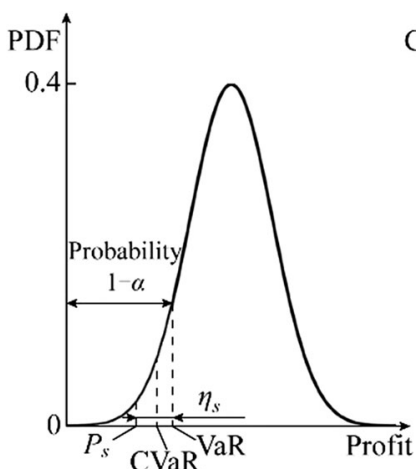

(a)

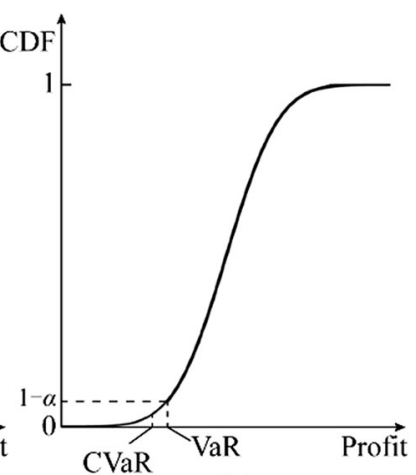

(b)
Fig. $3 \mathrm{VaR}$ and $\mathrm{CVaR}$ illustration on profit distribution 


\subsection{Assumptions}

1) Generation company is assumed to have no bilateral agreement and to trade energy only in the DA market. It does not participate in the balancing market by bidding up-regulation and down-regulation power; however, is obliged to exchange power with balancing market in case of a deviation from its DA power bid.

2) This paper aims to evaluate benefits of coordination and observe how thermal units react to wind power generation deviations. In order to see this explicitly, generation company is assumed to have forecasted DA and imbalance prices (i.e., MCP and SMP) and use them as direct inputs into its profit maximization problem. Forecasting MCP and SMP in electricity markets similar to the one in Turkey is a difficult task and is out of the scope of this paper. However, it should be mentioned that this study utilizes the output of a previous work which estimates MCP and SMP probabilistically based on artificial neural networks [19].

3) Given the estimated market prices for each hour, generation company is assumed to be a price taker which ensures acceptance of its bids to the DA market for each hour by bidding low prices. Thus, generation company is only concerned about the amount of power in DA market bidding, not the price.

4) Wind power scenarios have finite values with certain probabilities of occurrence, and sum of probabilities of these different scenarios is equal to 1 .

5) Hourly DA wind power forecast errors are assumed to have a normal distribution. Expected value and standard deviation of wind power forecast are available.

6) Generation company is assumed to degrade the system balance for all hours in case of a deviation, i.e., when generation company is short, the system is long or vice-versa. Hence, generation company is paid with the imbalance-up price for its excess generation sold to the balancing market and buys deficit power with imbalance-down price from balancing market.

7) System constraints such as spinning and non-spinning reserves for primary and secondary frequency control are not considered in the formulation.

8) Operating cost of wind power generation is assumed to be zero. Outputs of wind power units are aggregated and represented as if there is single wind power plant.

\subsection{Objective function}

The main objective of generation company's windthermal generation coordination problem is to maximize its total expected profit $P_{\mathrm{E}, \text { tot }}$ in Turkish DA market, as shown in (4).

$\max _{\left(P_{t g w}^{b i d}, P_{t s g}, u_{g}, \Delta_{t s}\right)} P_{\mathrm{E}, \mathrm{tot}}=\sum_{t=1}^{N_{T}} \sum_{s=1}^{N_{\mathrm{S}}} \pi_{s}\left(R_{t g w}-\sum_{g=1}^{N_{G}} C_{t s g}+P_{\mathrm{I}, t s}\right)$

where $t$ denotes index for bidding period; $w$ and $g$ denote indices for wind plant and thermal unit, respectively; $N_{T}$ and $N_{G}$ denote duration of period (hour) and number of units, respectively; $P_{t g w}^{b i d}$ denotes optimum DA bid at $t$ for coordinated generation; $P_{t s g}$ denotes power produced by thermal unit $g$ at time $t$ for scenario $s$; $u_{t g}$ denotes UC status of thermal unit $g$ at time $t, 1$ means on, 0 means off; $\Delta_{t s}$ denotes net imbalance power at time $t$ for scenario $s ; R_{\text {tgw }}$ denotes revenue from coordinated wind-thermal generation DA bid; $C_{t s g}$ denotes thermal generation cost of thermal unit $g$ at time $t$ for scenario $s ; P_{\mathrm{I}, t s}$ denotes imbalance penalty at time $t$ for scenario $s$.

Revenue of generation company results from the total energy sold to the DA market and the excess energy sold to the balancing market. The first and the second stage decision variables of the objective function are given in parenthesis. Note that the first stage decision variables the DA power bid $P_{t g w}^{b i d}$ and thermal unit status $u_{t g}-$ are independent of wind power scenarios, while the second stage decision variables - the thermal unit dispatch $P_{t s g}$ and the imbalance power $\Delta_{t s}-$ are dependent on the realization of wind power scenario.The terms within the brackets in (4) refer to per scenario revenue of the DA bid, total cost of thermal generation and imbalance penalty, respectively. These three terms can be expressed as follows:

$$
\begin{aligned}
& R_{\text {tgw }}=\rho_{t}^{d a} P_{t g w}^{b i d} \quad \forall t \\
& C_{t s g}=C_{\mathrm{Fg}} u_{t g}\left(a_{g} P_{t s g}^{2}+b_{g} P_{t s g}+c_{g}\right)+ \\
& \max \left\{0, C_{\mathrm{Sg} g}\left(u_{t g}-u_{t-1, g}\right)\right\} \quad \forall t, s, g
\end{aligned}
$$

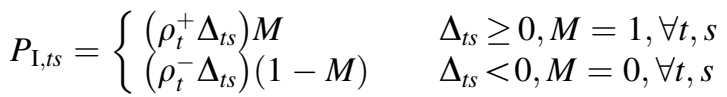

where $\rho_{t}^{d a}$ denotes DA market price at time; $\rho_{t}^{+}, \rho_{t}^{-}$denote imbalance-up and imbalance-down prices at time $t ; C_{\mathrm{Fg}}$ denotes fuel cost of thermal unit $g ; a_{g}, b_{g}, c_{g}$ denote heat rate curve parameters of unit $g ; C_{\mathrm{S} g}$ denotes start-up cost of thermal unit $g ; M$ denotes a binary variable.

As seen from (5), DA revenue is dependent on the power bid $P_{t g w}^{b i d}$ and the DA price $\rho_{t}^{d a}$. Total cost function of the thermal generation in (6) contains fuel cost of generation type, generation cost function and start-up cost of the unit. Thermal generation cost function is a quadratic function 
which causes nonlinearity in the objective function. Imbalance penalty function $P_{\mathrm{I}, t s}$ is modeled with a binary variable $M$ in (7). In this paper, an equivalent linear formulation proposed in [3] is used to eliminate the binary variable in order to obtain computational simplicity and efficiency in the solution. This linear formulation which is presented in (8) is descended from the decomposition of energy imbalance $\Delta_{t s}$ into summation of positive and negative imbalances $\Delta_{t s}^{-}$and $\Delta_{t s}^{+}$, respectively. Since the imbalance penalty, regardless of positive or negative, opposes to the maximization of profit, the optimization problem tends to minimize the imbalance penalty function. Therefore, without a necessity of $M$, the optimal solution is guaranteed with one of the variables $\Delta_{t s}^{-}$or $\Delta_{t s}^{+}$is zero since $\rho_{t}^{+} \leq \rho_{d a}$ and $\rho_{t}^{-} \geq \rho_{d a}$. Hence, thermal UC status $u_{t g}$ is the only integer variable in the problem.

$P_{\mathrm{I}, t s}=\rho_{t s}^{+} \Delta_{t s}^{+}-\rho_{t s}^{-} \Delta_{t s}^{-} \quad \forall t, s$

where $\Delta_{t s}^{+}$and $\Delta_{t s}^{-}$denote imbalance-up and imbalancedown power at time $t$ for scenario $s$.

The introduced coordinated objective function aims to maximize the expected profit excluding the risk. Risk assessment is crucial for the evaluation of the trade-off between profit and risk in stochastic problems. CVaR is included as a risk measurement term in the objective function given in (9). CVaR term is multiplied by a weighting factor - risk aversion parameter $\beta \in[0, \infty)$ - in order to simulate the effect of risk averse behavior on the expected profit and $\mathrm{CVaR}$.

$\max _{\left(P_{t g w}^{b i d}, P_{t s g}, u_{t g}, \Delta_{t s}^{+}, \Delta_{t s}^{-}\right)} P_{\mathrm{E}, \mathrm{tot}}+\beta \cdot C V a R_{\alpha}$

\subsection{Constraints}

There are various types of constraints that can be added to the wind-thermal generation coordination problem. Detailed description on system constraints, emission constraints, crew and other constraints are given in [20]. In this paper, bidding, market and thermal constraints are embedded in the problem formulation.

\subsubsection{Bidding constraints}

Bidding constraints are separately defined for stochastic and deterministic bidding approach for coordinated generation as well as uncoordinated generation.

1) Stochastic wind-thermal coordinated bidding

The amount of power bided to DA market must be within maximum and minimum wind power generation plus generation limits of thermal generators which are available (on) at that hour. Equation (10) presents the bidding constraint.
$P_{t s w}^{\min }+\sum_{g=1}^{N_{G}} P_{t s g}^{\min } u_{t g} \leq P_{t g w}^{b i d} P_{t s w}^{\max }+\sum_{g=1}^{N_{G}} P_{t s g}^{\max } u_{t g} \quad \forall t$

where $P_{t s w}^{\min }$ and $P_{t s w}^{\max }$ denote the minimum and maximum wind power generations at time $t$ for scenario $s ; P_{t s g}^{\min }$ and $P_{t s g}^{\max }$ denote the minimum and maximum power outputs of thermal unit $g$ at time $t$ for scenario $s$.

2) Deterministic wind-thermal coordinated bidding

Power bid is within the limits between the possible maximum and minimum generation limits of thermal generators available at that hour. The amount of power bid for wind generation is determined as if wind power forecast is certain. Equation (11) illustrates the deterministic windthermal bidding constraint:

$P_{t w}^{\exp }+\sum_{g=1}^{N_{G}} P_{t s g}^{\min } u_{t g} \leq P_{t g w}^{b i d} \leq P_{t w}^{\exp }+\sum_{g=1}^{N_{G}} P_{t s g}^{\max } u_{t g} \quad \forall t$

where $P_{t w}^{\exp }$ denotes expected wind power generation at time $t$.

3) Stochastic wind-thermal uncoordinated bidding

Power bids of thermal and wind units are determined separately as in (12) and (13).

$\sum_{g=1}^{N_{G}} P_{t g}^{\min } u_{t g} \leq P_{t g}^{b i d} \leq \sum_{g=1}^{N_{G}} P_{t g}^{\max } u_{t g} \quad \forall t$

$P_{t s w}^{\min } \leq P_{t w}^{b i d} \leq P_{t s w}^{\max } \quad \forall t$

where $P_{t g}^{b i d}$ and $P_{t w}^{b i d}$ denote optimum DA bid and optimum DA power bid at time $t$ for uncoordinated thermal generation, respectively; $P_{t g}^{\min }$ and $P_{t g}^{\max }$ denote the minimum and maximum power outputs of thermal unit at time $t$.

\subsubsection{Imbalance price constraints}

Double price mechanism which is practiced by Turkish balancing market is presented below for the supplier side.

$$
\begin{cases}\rho_{t}^{s m p} \leq \rho_{t}^{d a} & \lambda>0, \forall t \\ \rho_{t}^{s m p} \geq \rho_{t}^{d a} & \lambda<0, \forall t\end{cases}
$$

where $\rho_{t}^{\text {smp }}$ denotes SMP at time $t ; \lambda$ denotes system direction.

1) When generation company's real-time generation is more than the amount of DA schedule, excess generation is sold to the balancing market with imbalance-up price $\rho_{t}^{+}$as in (15). 
$\rho_{t}^{+}= \begin{cases}\min \left\{\rho_{t}^{d a}, \rho_{t}^{s m p}\right\}=\rho_{t}^{s m p} & \lambda>0, \forall t \\ \max \left\{\rho_{t}^{d a}, \rho_{t}^{s m p}\right\}=\rho_{t}^{d a} & \lambda<0, \forall t\end{cases}$

2) When generation company's real-time generation is less than the amount of DA power schedule, generation deficit is bought from balancing market with imbalance down price $\rho_{t}^{-}$as in (16).

$\rho_{t}^{-}= \begin{cases}\min \left\{\rho_{t}^{d a}, \rho_{t}^{s m p}\right\}=\rho_{t}^{d a} & \lambda>0, \forall t \\ \max \left\{\rho_{t}^{d a}, \rho_{t}^{s m p}\right\}=\rho_{t} \operatorname{smp} & \lambda<0, \forall t\end{cases}$

Since it is assumed that generation company degrades the system balance for all hours, constraint on imbalance prices can be summarized as in (17).

$\rho_{t}^{+} \leq \rho_{t}^{d a} \leq \rho_{t}^{-} \quad \forall t$

\subsubsection{Imbalance power constraints}

Imbalance power is equal to the difference between DA power schedule and real-time generation of generation company. Either $\Delta_{t s}^{+}$or $\Delta_{t s}^{-}$is equal to zero for all $t$ and $s$ due to nature of the optimization problem. Equation (18) presents the imbalance power constraint.

$\Delta_{t s}=P_{t s w}+\sum_{g=1}^{N_{G}} P_{t s g}-P_{t g w}^{b i d}=\Delta_{t s}^{+}-\Delta_{t s}^{-} \quad \forall t, s$

\subsubsection{Thermal unit constraints}

Thermal unit constraints considered in the paper are generation, ramp-up and ramp-down power and minimumup and minimum-down time limits.

\section{1) Unit's ramp-up and ramp-down capacity constraints}

Due to machinery limits, electrical output of a thermal unit cannot change more than a certain amount over a period of time. Generation of thermal unit for successive hours is bounded by ramp-up and ramp-down constraints, as shown in (19).

$-\bar{R}_{D g} \leq P_{t s g}-P_{t-1, s g} \leq \bar{R}_{U g} \quad \forall t, s, g$

where $\bar{R}_{U g}$ and $\bar{R}_{D g}$ denote the maximum ramp-up rate limit and the maximum ramp-down rate limit of thermal unit $g$, respectively.

\section{2) Generation constraints}

Generation constraints are defined as the minimum and maximum feasible generation capacity of an operating thermal unit. In addition, ramp-up and ramp-down constraints related with the units should be taken into account, as shown in (20)-(22).

$P_{t s g}^{\min } u_{t g} \leq P_{t s g} \leq P_{t s g}^{\max } u_{t g} \quad \forall t, s, g$
$P_{t s g}= \begin{cases}\min \left\{P_{t-1, s g}+\bar{R}_{U g}, \bar{P}_{g}\right\} & u_{t g}=u_{t-1, g}=1, \forall t, s, g \\ \min \left\{P_{g}+R_{u g}, P_{g}\right\} & u_{t g}=1, u_{t-1, g}=0, \forall t, s, g\end{cases}$

$P_{t s g}= \begin{cases}\min \left\{P_{t-1, s g}+\bar{R}_{D g}, \underline{P}_{g}\right\} & u_{t g}=u_{t-1, g}=1, \forall t, s, g \\ \underline{P}_{g} & u_{t g}=1, u_{t-1, g}=0, \forall t, s, g\end{cases}$

where $\bar{P}_{g}$ and $\underline{P}_{g}$ denote the maximum and the minimum thermal power output limits of thermal unit $g$.

3) Minimum-up and minimum-down time constraints

Once a unit is running, it cannot be shut down immediately. Likewise, the off units cannot be started immediately. Time requirement for a thermal unit to be turned off and on is defined as minimum-up and minimum-down times as given in (23) and (24), respectively.

$\begin{array}{ll}\left(T_{t-1, g}^{u p}-T_{\text {min }, g}^{u p}\right)\left(u_{t-1, g}-u_{t g}\right) \geq 0 & \forall t, g \\ \left(T_{t-1, g}^{d n}-T_{\text {min }, g}^{d n}\right)\left(u_{t g}-u_{t-1, g}\right) \geq 0 & \forall t, g\end{array}$

where $T_{t-1, g}^{u p}$ and $T_{t-1, g}^{d n}$ denote the time that thermal unit $g$ has been up and the time that thermal unit $g$ has been down at time $t$, respectively; $T_{\min , g}^{u p}$ and $T_{\text {min, } g}^{d n}$ denote the minimum-up time and the minimum-down time of thermal unit $g$, respectively.

\section{Solution algorithm}

The solution algorithm for the wind-thermal coordination, which is developed in MATLAB environment, is presented in a flowchart in Fig. 4. Dynamic programming (DP) is used in order to eliminate the mixed-integer nature of the problem formulation and find the optimum UC of thermal units.

In the first stage of the solution algorithm, $t$ and $k$, which denote time and number of feasible previous transitions, respectively, are initialized. $t$ is initialized as 1 since it is the beginning of the scheduling period and $k$ is initialized as 1 due to there is only one feasible previous state at $t=1$ (i.e., operating conditions of the units in the last hour of the previous day). Also, at this stage of the algorithm, the problem is fed with market, wind and thermal unit data. These include:

1) Market data: forecasted DA clearing price, imbalanceup and imbalance-down prices.

2) Wind power forecast data: expected wind power and standard deviation.

3) Thermal unit data: number of units, unit generation capacity, ramp-up/down limits, start-up costs, initial on/off durations, minimum-up/down time, fuel price, and cost coefficients for each unit. 


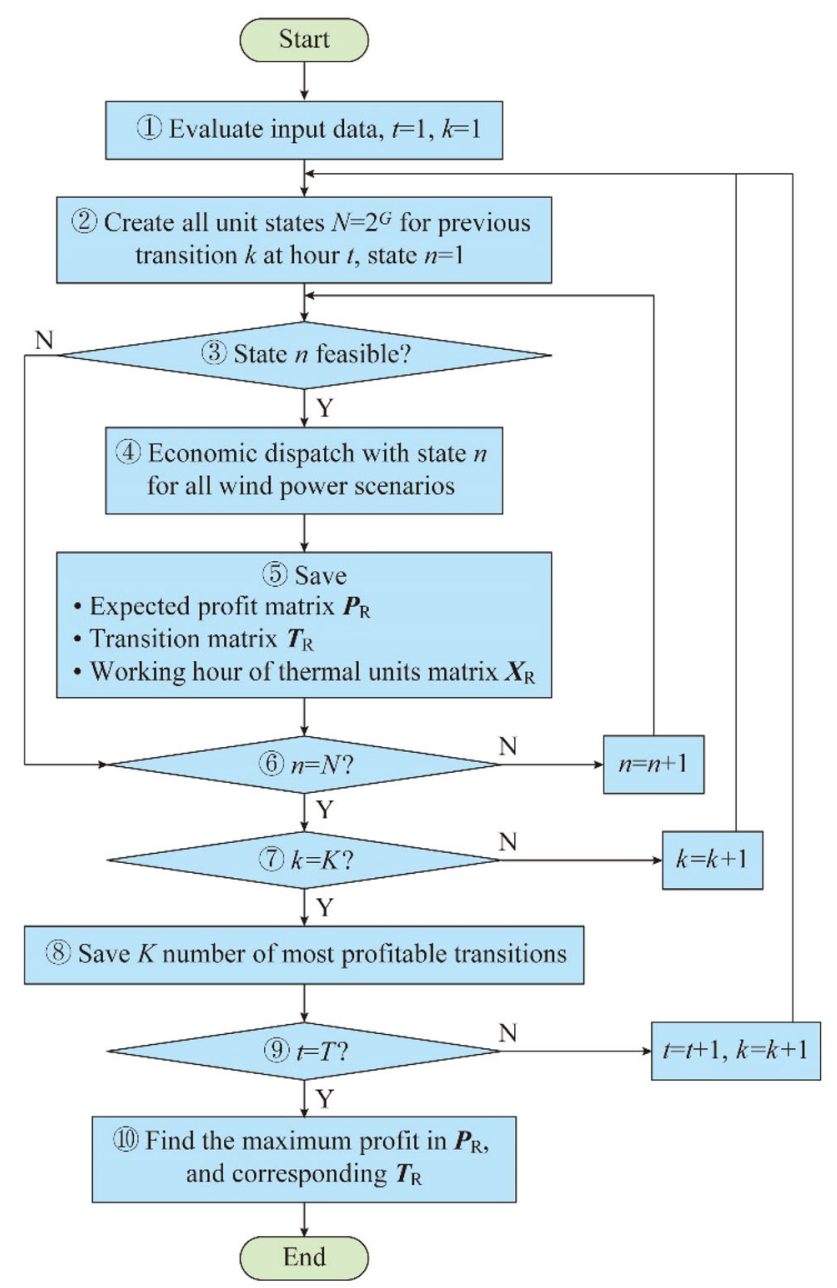

Fig. 4 Solution algorithm for wind-thermal coordination

In the second stage of the algorithm, all possible $N=2^{G}$ ( $G$ is the number of thermal units) thermal UC states for each previous transition $k$ and hour $t$ are created with complete enumeration. However, only limited number of statuses is recorded to reduce the computational time, as discussed below.

Whether transition $k$ from previous hour to current hour for state $n$ is feasible or not is checked with minimum-up and minimum-down constraints in the third stage of the algorithm. For this purpose, on/off data of thermal units in current state $n$ are compared to data stored in $\boldsymbol{X}_{\mathrm{R}}$ (working hour of thermal unit matrix) which keeps the duration that thermal units have been on or off until that hour. Then, $\boldsymbol{X}_{\mathrm{R}}$ is updated for the next hour if transition to current state is feasible. Infeasible transitions are not stored in the memory.

In the fourth stage of the algorithm, ED with thermal units which are on is conducted for each wind power scenario. Expected profits and corresponding optimum transition sub-paths are saved in $\boldsymbol{P}_{\mathrm{R}}$ (profit matrix) and $\boldsymbol{T}_{\mathrm{R}}$ (transition matrix), respectively.

Obtaining an optimal solution for this problem is not easy. The size of the transition matrix $\boldsymbol{T}_{\mathrm{R}}$ increases with $t$ through the scheduling horizon. As mentioned before, for $G$ number of thermal units there are $2^{G}$ possible states. Assume that all state transitions are feasible, for $T$ hours of scheduling horizon, size of $\boldsymbol{T}_{\mathrm{R}}, K$, becomes $\left(2^{G}\right)^{T}$. In order to reduce the computational effort, time and program memory, at each hour not all but $K$ number of most profitable states are saved in $\boldsymbol{P}_{\mathrm{R}}, \boldsymbol{T}_{\mathrm{R}}$ and $\boldsymbol{X}_{\mathrm{R}}$ in the fifth, sixth and seventh stages. This approach may result in a suboptimal solution. In the eighth stage of the problem, the algorithm decides on the number of transitions $K$ to be saved for the next hour. Whether the DP reaches to the last hour in the scheduling period is checked in the ninth stage with the counter $t$. In the final stage, the suboptimal transition path (thus the UC schedule) is found by the transition path saved in $\boldsymbol{T}_{\mathrm{R}}$ that corresponds to the maximum profit in $\boldsymbol{P}_{\mathrm{R}}$.

\section{Numerical studies and results}

Effects of coordination and bidding strategy on the expected profit and CVaR are evaluated in a 24-hour scheduling problem for different scenarios. Problems are solved in a computer that has Intel Core i5 processor with $2.60 \mathrm{GHz}$ and $8 \mathrm{~GB}$ memory. Not all but $K$ number of most profitable states are saved in each stage of the DP in order to reduce the computational size and time. Nevertheless, this may result in a suboptimal solution since not all the integer combinations are searched by DP. In order to test the optimality of solution, expected profit and execution time are compared under different $K$ values. It is found that the value of 8 for $K$ provides the best balance between optimal solution and execution time.

The generation company considered in the numerical studies is assumed to own two thermal units and a wind farm. The capacity of the wind farm is $180 \mathrm{MW}$, while the total installed capacity of thermal plants is $90 \mathrm{MW}$. Market prices and wind power forecasts are inputs to the proposed model. Thermal unit data are given in Table 1 , where $u_{i n i}$ represents the number of hours that unit has been on.

It is assumed that hourly wind power forecast error lies within a normal distribution fashion similar to $[5,13,21,22]$ and an increasing standard deviation in the later hours of the day. Instead of dealing with a continuous PDF, discrete variables which are called scenarios that could represent continuous variables are generally preferred [12, 19, 23]. In order to find a good approximation for the wind power forecast, large number of scenarios is needed to cover the probability space. On the other hand, 
Table 1 Thermal unit data

\begin{tabular}{|c|c|c|c|c|c|c|c|}
\hline Unit No. & $\underline{P}_{g}(\mathrm{MW})$ & $\bar{P}_{g}(\mathrm{MW})$ & $T_{\min , g}^{u p}$ (hour) & $T_{\min , g}^{d n}$ (hour) & $u_{i n i}$ (hour) & \multicolumn{2}{|c|}{ Fuel cost $(\$ / M b t u)$} \\
\hline 1 & 5 & 45 & 4 & 2 & -2 & 1.0 & \\
\hline 2 & 5 & 45 & 1 & 1 & -2 & 1.0 & \\
\hline Unit No. & $\bar{R}_{U g}(\mathrm{MW})$ & $\bar{R}_{D g}(\mathrm{MW})$ & $a_{g}(\mathrm{Mbtu})$ & $b_{g}(\mathrm{Mbtu} / \mathrm{MW})$ & $c_{g}(\mathrm{M}$ & & $C_{\mathrm{S} g}(\$)$ \\
\hline 1 & 10 & 10 & 85.51 & 70.86 & 0.19 & & 100 \\
\hline 2 & 40 & 40 & 89.34 & 78.23 & 0.23 & & 0 \\
\hline
\end{tabular}

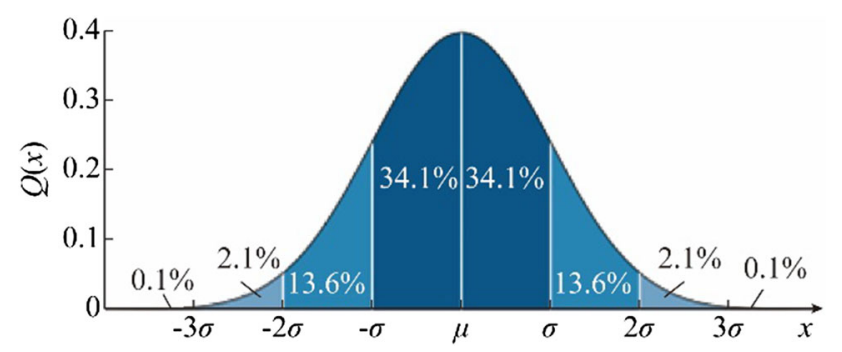

Fig. 5 Normal PDF of wind power forecast and confidence intervals with respect to $\sigma$

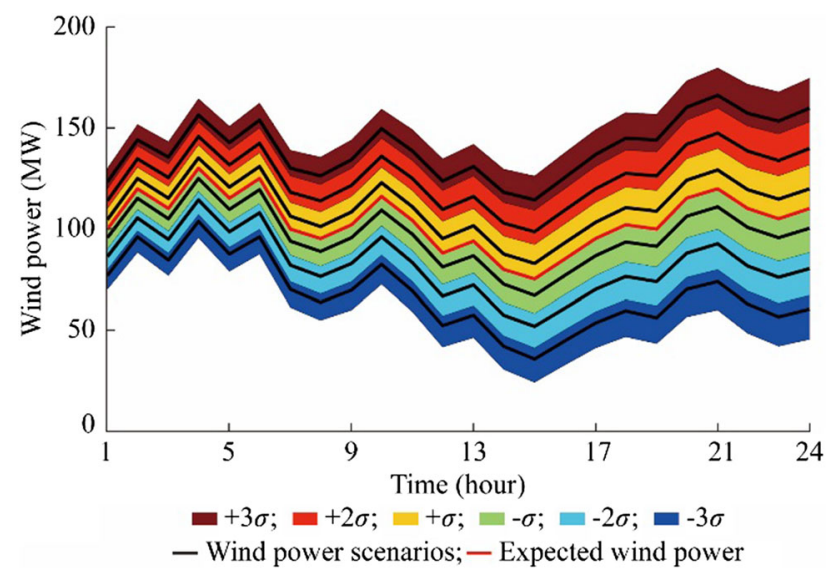

Fig. 6 Wind power scenarios for every $\sigma$ interval

there is a trade-off between number of sample scenarios and the computational complexity. For the scenario studies, PDF of the wind power forecast error, denoted as $Q(x)$ in Fig. 5, for each hour is divided into six confidence intervals with a standard deviation of $\sigma$ around the expected value $\mu$ in the $x$-axis between $[-3 \sigma,+3 \sigma]$ so as to find representative scenarios. The interval $[-3 \sigma,+3 \sigma]$ spans the $99.74 \%$ of the total area in PDF. This can be interpreted as the probability of the wind power outcome in the next day lies within this interval with $99.74 \%$ of chance. Therefore, values which deviate more than $3 \sigma$ from the expected wind power forecast value are ignored to reduce the computational burden and execution time. In Fig. 6, six
Table 2 Market data

\begin{tabular}{|c|c|c|c|}
\hline Time (hour) & $\rho_{t}^{d a}(\$ / \mathrm{MWh})$ & $\rho_{t}^{+}(\$ / \mathrm{MWh})$ & $\rho_{t}^{-}(\$ / \mathrm{MWh})$ \\
\hline 1 & 74 & 40 & 100 \\
\hline 2 & 86 & 55 & 120 \\
\hline 3 & 75 & 65 & 112 \\
\hline 4 & 95 & 50 & 101 \\
\hline 5 & 70 & 35 & 88 \\
\hline 6 & 95 & 50 & 118 \\
\hline 7 & 77 & 72 & 80 \\
\hline 8 & 85 & 46 & 128 \\
\hline 9 & 75 & 64 & 114 \\
\hline 10 & 72 & 36 & 102 \\
\hline 11 & 76 & 56 & 90 \\
\hline 12 & 70 & 62 & 82 \\
\hline 13 & 82 & 68 & 94 \\
\hline 14 & 88 & 83 & 101 \\
\hline 15 & 92 & 78 & 104 \\
\hline 16 & 95 & 91 & 124 \\
\hline 17 & 77 & 58 & 93 \\
\hline 18 & 75 & 71 & 87 \\
\hline 19 & 70 & 50 & 81 \\
\hline 20 & 75 & 48 & 84 \\
\hline 21 & 82 & 72 & 88 \\
\hline 22 & 88 & 75 & 114 \\
\hline 23 & 94 & 62 & 120 \\
\hline 24 & 80 & 60 & 102 \\
\hline
\end{tabular}

representative wind power scenarios for different $\sigma$ intervals are drawn in black lines.

The six different scenarios investigated throughout this paper are uncoordinated wind-thermal generation (scenario 1 , corresponds to $-3 \sigma$ ), coordinated wind-thermal generation with risk attitude parameter $\beta$ of $0,0.1,0.5$ and 1.0 (scenarios 2, 3, 4, 5, correspond to $-2 \sigma,-\sigma, \sigma, 2 \sigma$ ), and coordinated wind-thermal generation with deterministic bidding (scenario 6, corresponds to $3 \sigma$ ). Market data and wind power forecast data considered in the study are presented in Table 2 and Table 3, respectively. Wind power 
Table 3 Wind power forecast data

\begin{tabular}{|c|c|c|}
\hline Time (hour) & $P_{t w}^{e x p}(\mathrm{MW})$ & $\sigma(\mathrm{MW})$ \\
\hline 1 & 100 & 10.00 \\
\hline 2 & 120 & 10.50 \\
\hline 3 & 110 & 11.00 \\
\hline 4 & 130 & 11.50 \\
\hline 5 & 115 & 12.00 \\
\hline 6 & 125 & 12.50 \\
\hline 7 & 100 & 13.00 \\
\hline 8 & 95 & 13.50 \\
\hline 9 & 102 & 14.00 \\
\hline 10 & 116 & 14.50 \\
\hline 11 & 104 & 15.00 \\
\hline 12 & 88 & 15.50 \\
\hline 13 & 94 & 16.00 \\
\hline 14 & 80 & 16.50 \\
\hline 15 & 75 & 17.00 \\
\hline 16 & 85 & 17.50 \\
\hline 17 & 95 & 18.00 \\
\hline 18 & 102 & 18.50 \\
\hline 19 & 100 & 19.00 \\
\hline 20 & 115 & 19.50 \\
\hline 21 & 120 & 20.00 \\
\hline 22 & 110 & 20.50 \\
\hline 23 & 105 & 21.00 \\
\hline 24 & 110 & 21.50 \\
\hline
\end{tabular}

generation data for scenarios 1-6, which are developed based on assumptions in Table 3 , are illustrated in Table 4.

$\mathrm{UC}$ results in Table 5 (U1 and $\mathrm{U} 2$ represent unit 1 and unit 2) ensure optimal trade-off between the expected profit and the risk under wind uncertainty under the ED solutions presented in Table 6.

The profit and $\mathrm{CVaR}$ values for each scenario are presented in Table 7. CVaR is calculated for $\alpha=0.98$ $\left(\mathrm{CVaR}_{0.98}\right)$ which equals to the value of lowest profitable scenario in the problem. In terms of expected profit and CVaR, coordinated wind-thermal generation with stochastic bidding is prominent to uncoordinated generation and coordinated generation with deterministic bidding. Expected profit of coordinated generation is $1.25 \%$ which is $0.5 \%$ higher than that of uncoordinated generation and coordinated deterministic bidding. Situation in $\mathrm{CVaR}$ is even more distinct for coordinated generation with $4.6 \%$ and $2.4 \%$ higher $\mathrm{CVaR}$ values compared to uncoordinated generation and coordinated deterministic bidding, respectively. Note that $\mathrm{CVaR}$ of uncoordinated thermal generation is equal to its expected profit as there is no stochasticity in thermal only generation.
Table 4 Wind power generation data for different scenarios

\begin{tabular}{|c|c|c|c|c|c|c|}
\hline \multirow[t]{2}{*}{ Time (hour) } & \multicolumn{6}{|c|}{ Wind power generation data (MW) } \\
\hline & 1 & 2 & 3 & 4 & 5 & 6 \\
\hline 1 & 76.92 & 86.22 & 95.42 & 104.58 & 113.78 & 123.08 \\
\hline 2 & 95.77 & 105.53 & 115.19 & 124.81 & 134.47 & 144.23 \\
\hline 3 & 84.61 & 94.84 & 104.96 & 115.04 & 125.16 & 135.39 \\
\hline 4 & 103.45 & 114.15 & 124.73 & 135.27 & 145.85 & 156.55 \\
\hline 5 & 87.29 & 98.46 & 109.50 & 120.50 & 131.54 & 142.71 \\
\hline 6 & 96.13 & 107.76 & 119.27 & 130.73 & 142.24 & 153.87 \\
\hline 7 & 69.98 & 82.07 & 94.04 & 105.96 & 117.93 & 130.02 \\
\hline 8 & 63.82 & 76.38 & 88.81 & 101.19 & 113.62 & 126.18 \\
\hline 9 & 69.66 & 82.69 & 95.58 & 108.42 & 121.31 & 134.34 \\
\hline 10 & 82.50 & 96.00 & 109.35 & 122.65 & 136.00 & 149.50 \\
\hline 11 & 69.34 & 83.31 & 97.12 & 110.88 & 124.69 & 138.66 \\
\hline 12 & 52.19 & 66.62 & 80.89 & 95.11 & 109.38 & 123.81 \\
\hline 13 & 57.03 & 71.92 & 86.66 & 101.34 & 116.08 & 130.97 \\
\hline 14 & 41.87 & 57.23 & 72.43 & 87.57 & 102.77 & 118.13 \\
\hline 15 & 35.71 & 51.54 & 67.20 & 82.80 & 98.46 & 114.29 \\
\hline 16 & 44.55 & 60.85 & 76.97 & 93.03 & 109.15 & 125.45 \\
\hline 17 & 53.40 & 70.16 & 86.74 & 103.26 & 119.84 & 136.60 \\
\hline 18 & 59.24 & 76.47 & 93.51 & 110.49 & 127.53 & 144.76 \\
\hline 19 & 56.08 & 73.77 & 91.28 & 108.72 & 126.23 & 143.92 \\
\hline 20 & 69.92 & 88.08 & 106.05 & 123.95 & 141.92 & 160.08 \\
\hline 21 & 73.77 & 92.39 & 110.82 & 129.18 & 147.61 & 166.23 \\
\hline 22 & 62.61 & 81.70 & 100.59 & 119.41 & 138.30 & 157.39 \\
\hline 23 & 56.45 & 76.01 & 95.36 & 114.64 & 133.99 & 153.55 \\
\hline 24 & 60.29 & 80.32 & 100.13 & 119.87 & 139.68 & 159.71 \\
\hline
\end{tabular}

Expected profit versus CVaR plot is given for all scenarios in Fig. 7. Uncoordinated bidding has the lowest expected profit and CVaR among all scenarios. For coordinated generation, as the risk averse behavior increases with $\beta ; C V a R_{0.98}$ increases as well but the expected profit decreases. The performance of coordinated deterministic bidding in terms of expected profit and $\mathrm{CVaR}$ is better than that of uncoordinated generation. Comparing scenario 1 and scenario 5 reveals that $4.6 \%$ increase in $\mathrm{CVaR}$ results in only $1.3 \%$ reduction in the expected profit. According to this trade-off between the expected profit and CVaR, generation company can choose its preference of risk before bidding in the DA market.

The risk on profit variability can be controlled at the cost of a small reduction in the expected profit. Figure 8 illustrates such a case for the coordinated wind-thermal generation with generation company's attitude towards risk. As the risk averse behavior increases with $\beta$, standard deviation of realized profits decreases. In Fig. 9, coordinated wind-thermal generation with $\beta=0$ is solved for different values of $K$ and expected profit and execution 
Table 5 UC statuses of thermal units

\begin{tabular}{|c|c|c|c|c|c|c|c|c|c|c|c|c|}
\hline \multirow[t]{3}{*}{ Time (hour) } & \multicolumn{12}{|c|}{ Thermal UC status } \\
\hline & \multicolumn{2}{|l|}{1} & \multicolumn{2}{|l|}{2} & \multicolumn{2}{|l|}{3} & \multicolumn{2}{|l|}{4} & \multicolumn{2}{|l|}{5} & \multicolumn{2}{|l|}{6} \\
\hline & $\mathrm{U} 1$ & $\mathrm{U} 2$ & $\mathrm{U} 1$ & $\mathrm{U} 2$ & $\mathrm{U} 1$ & $\mathrm{U} 2$ & $\mathrm{U} 1$ & $\mathrm{U} 2$ & U1 & $\mathrm{U} 2$ & $\mathrm{U} 1$ & $\mathrm{U} 2$ \\
\hline 1 & 1 & 0 & 1 & 0 & 1 & 0 & 1 & 0 & 1 & 0 & 1 & 0 \\
\hline 2 & 1 & 0 & 1 & 1 & 1 & 1 & 1 & 1 & 1 & 1 & 1 & 0 \\
\hline 3 & 1 & 0 & 1 & 0 & 1 & 0 & 1 & 0 & 1 & 0 & 1 & 0 \\
\hline 4 & 1 & 1 & 1 & 1 & 1 & 1 & 1 & 1 & 1 & 1 & 1 & 1 \\
\hline 5 & 1 & 0 & 1 & 0 & 1 & 0 & 1 & 0 & 1 & 0 & 1 & 0 \\
\hline 6 & 1 & 1 & 1 & 1 & 1 & 1 & 1 & 1 & 1 & 1 & 1 & 1 \\
\hline 7 & 1 & 0 & 1 & 0 & 1 & 0 & 1 & 0 & 1 & 0 & 1 & 0 \\
\hline 8 & 1 & 0 & 1 & 1 & 1 & 1 & 1 & 1 & 1 & 1 & 1 & 0 \\
\hline 9 & 1 & 0 & 1 & 0 & 1 & 0 & 1 & 0 & 1 & 0 & 1 & 0 \\
\hline 10 & 0 & 0 & 1 & 0 & 1 & 0 & 1 & 1 & 1 & 1 & 1 & 0 \\
\hline 11 & 0 & 0 & 1 & 0 & 1 & 0 & 1 & 0 & 1 & 0 & 0 & 0 \\
\hline 12 & 0 & 0 & 1 & 0 & 1 & 0 & 1 & 0 & 1 & 0 & 0 & 0 \\
\hline 13 & 1 & 0 & 1 & 0 & 1 & 0 & 1 & 1 & 1 & 1 & 1 & 0 \\
\hline 14 & 1 & 0 & 1 & 1 & 1 & 1 & 1 & 1 & 1 & 1 & 1 & 1 \\
\hline 15 & 1 & 1 & 1 & 1 & 1 & 1 & 1 & 1 & 1 & 1 & 1 & 1 \\
\hline 16 & 1 & 1 & 1 & 1 & 1 & 1 & 1 & 1 & 1 & 1 & 1 & 1 \\
\hline 17 & 1 & 0 & 1 & 0 & 1 & 0 & 1 & 0 & 1 & 0 & 1 & 0 \\
\hline 18 & 1 & 0 & 1 & 0 & 1 & 0 & 1 & 0 & 1 & 0 & 1 & 0 \\
\hline 19 & 1 & 0 & 1 & 0 & 1 & 0 & 1 & 0 & 1 & 0 & 1 & 0 \\
\hline 20 & 1 & 0 & 1 & 0 & 1 & 0 & 1 & 0 & 1 & 0 & 1 & 0 \\
\hline 21 & 1 & 0 & 1 & 0 & 1 & 0 & 1 & 0 & 1 & 0 & 1 & 0 \\
\hline 22 & 1 & 1 & 1 & 1 & 1 & 1 & 1 & 1 & 1 & 1 & 1 & 1 \\
\hline 23 & 1 & 1 & 1 & 1 & 1 & 1 & 1 & 1 & 1 & 1 & 1 & 1 \\
\hline 24 & 1 & 0 & 1 & 1 & 1 & 1 & 1 & 1 & 1 & 1 & 1 & 0 \\
\hline
\end{tabular}

time results are presented. According to results, optimality of the solution changes with $K$; expected profit increases as the value of $K$ increases due to the larger search space scanned with DP. For $K>4$, significant increment in the expected profit is not observed; hence, for this specific problem one can make a tradeoff between $K$ and the execution time.

\section{Conclusion}

In this paper, a two-stage stochastic programming model for wind-thermal coordination is proposed. The problem considers a generation company participating in Turkish DA electricity market with its thermal and wind generation units. The main objective of the generation company is to determine the optimum power for the DA market to maximize its expected profit in the first stage while controlling risks associated with possible realizations of wind power output in the second stage. Based on this objective, generation company obtains the most suitable hourly generation schedule and optimal bids under CVaR consideration. Comparative scenario studies are investigated to illustrate the performance of bidding strategies and benefits of the coordination. Results indicate that the wind-thermal generation coordination could significantly contribute to profit of generation company by reducing the imbalance penalty charged by the balancing market. Coordination also steers thermal units to commit more often to balance realtime generation deviations from the DA bid caused by the wind uncertainty. Thus, ramp limits, start-up cost, minimum-up times, minimum-down time and generation capacity of thermal units can greatly affect the benefit of coordination. To determine hourly power bid to market, generation company should not compute optimal thermal and wind power bids separately but aggregate wind and thermal power bids in order to have higher profit. Windthermal coordination not only improves expected profits but also substantially increases the CVaR. The imbalance penalty for any discrepancy between the DA schedule and 
Table 6 Amount of DA power bid

\begin{tabular}{|c|c|c|c|c|c|c|}
\hline \multirow[t]{2}{*}{ Time (hour) } & \multicolumn{6}{|c|}{ DA power bid (MW) } \\
\hline & 1 & 2 & 3 & 4 & 5 & 6 \\
\hline 1 & 112.9 & 110.4 & 109.6 & 102.9 & 100.4 & 108.4 \\
\hline 2 & 140.2 & 162.8 & 160.4 & 154.5 & 153.1 & 145.0 \\
\hline 3 & 120.0 & 130.0 & 129.8 & 119.6 & 119.6 & 125.0 \\
\hline 4 & 217.1 & 211.9 & 210.2 & 204.1 & 196.6 & 201.3 \\
\hline 5 & 135.5 & 145.5 & 145.5 & 144.5 & 134.5 & 130.0 \\
\hline 6 & 202.0 & 199.4 & 197.8 & 186.1 & 186.1 & 196.3 \\
\hline 7 & 122.3 & 131.0 & 131.0 & 119.0 & 119.0 & 116.3 \\
\hline 8 & 123.8 & 143.6 & 143.6 & 139.2 & 131.2 & 130.0 \\
\hline 9 & 110.6 & 110.6 & 107.7 & 94.7 & 94.7 & 117.0 \\
\hline 10 & 127.7 & 127.7 & 125.3 & 132.7 & 119.4 & 121.0 \\
\hline 11 & 110.9 & 115.9 & 115.9 & 112.1 & 102.1 & 104.0 \\
\hline 12 & 80.9 & 95.0 & 85.9 & 73.6 & 67.2 & 88.0 \\
\hline 13 & 116.3 & 116.3 & 111.7 & 119.0 & 108.2 & 109.0 \\
\hline 14 & 118.6 & 133.3 & 128.4 & 115.0 & 107.7 & 126.1 \\
\hline 15 & 147.6 & 147.8 & 143.5 & 130.5 & 125.7 & 139.8 \\
\hline 16 & 142.1 & 150.9 & 139.6 & 134.6 & 134.0 & 166.3 \\
\hline 17 & 128.3 & 128.2 & 121.7 & 111.7 & 95.2 & 120.0 \\
\hline 18 & 108.5 & 108.5 & 108.5 & 84.2 & 80.4 & 117.0 \\
\hline 19 & 113.7 & 113.7 & 113.7 & 106.3 & 96.3 & 105.0 \\
\hline 20 & 139.0 & 139.0 & 129.0 & 129.0 & 121.1 & 130.0 \\
\hline 21 & 158.8 & 154.2 & 144.2 & 135.8 & 125.8 & 149.6 \\
\hline 22 & 166.7 & 171.7 & 156.0 & 142.6 & 137.1 & 176.1 \\
\hline 23 & 193.7 & 177.6 & 174.0 & 154.6 & 146.5 & 184.1 \\
\hline 24 & 125.1 & 149.9 & 149.9 & 134.6 & 130.1 & 135.0 \\
\hline
\end{tabular}

Table 7 Profit analysis of scenario studies

\begin{tabular}{lrr}
\hline Scenario & $\operatorname{CVaR}_{0.98}(\$)$ & \multicolumn{1}{c}{$P_{\mathrm{E}, \mathrm{tot}}(\$)$} \\
\hline Uncoordinated thermal & 3731.21 & 3731.21 \\
Uncoordinated wind & 115481.59 & 197705.77 \\
Scenario 1 & 119212.81 & 201436.98 \\
Scenario 2 & 124641.41 & 203945.83 \\
Scenario 3 & 125893.38 & 203824.79 \\
Scenario 4 & 130151.08 & 202669.44 \\
Scenario 5 & 132088.57 & 201288.32 \\
Scenario 6 & 121738.92 & 202895.95
\end{tabular}

the real-time delivery may force generation company to accept more risk averse decisions to increase CVaR. The $\mathrm{CVaR}$ criterion maximizes the expected profits of the lowest possible wind power scenarios which lessens the chance of having low profits. Generation company can effectively control the trade-off between $\mathrm{CVaR}$ and expected profit with the proposed model. Small reduction in the expected profit can result in high growth in CVaR. Hence, generation company determines its generation

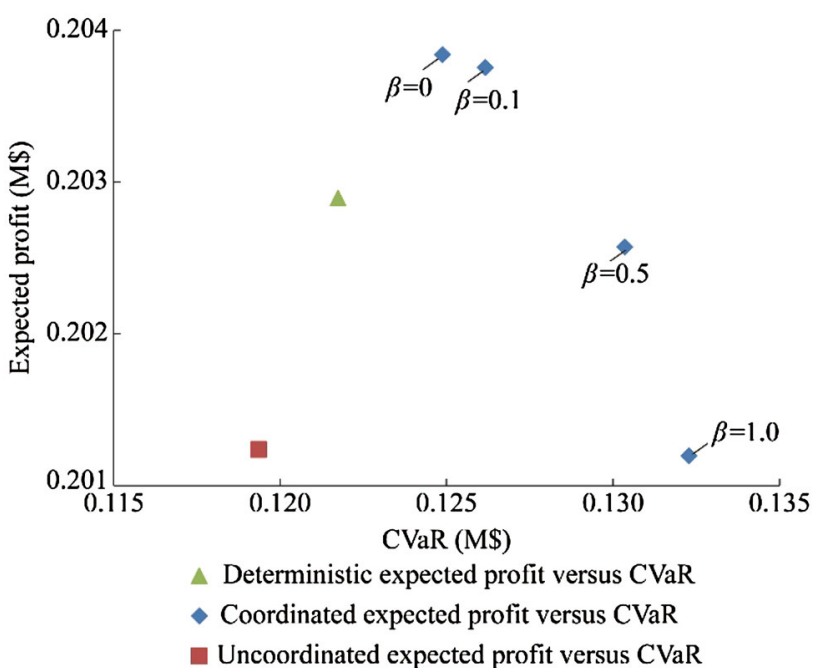

Fig. 7 Expected profit versus $\mathrm{CVaR}$

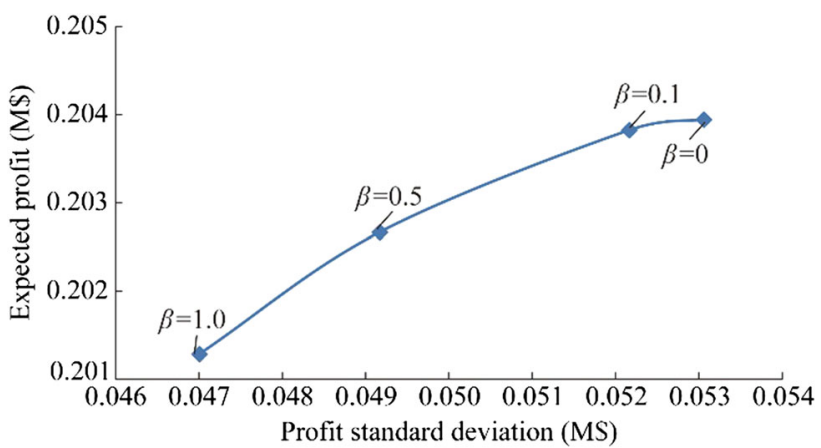

Fig. 8 Expected profit versus standard deviation

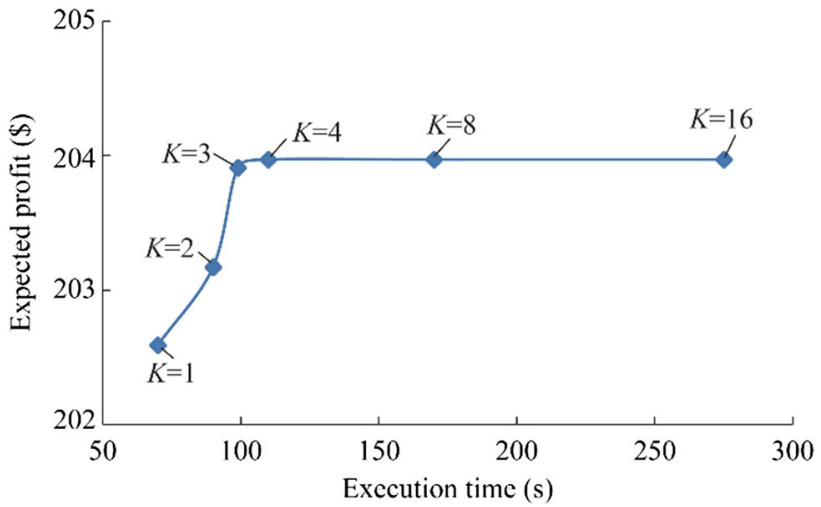

Fig. 9 Expected profit versus execution time with respect to $K$

scheduling and DA bidding according to associated risk preference.

In this study, the DA market prices are assumed to be forecasted deterministically. Future studies might include effects of stochastic DA price forecasting on the results. Analysis can be further scaled up with other types of generation such as hydro pumped-storage. Impact of 
greenhouse emission caps on electricity generation facilities which are likely to be imposed in near future by governments aiming to comply with international agreements can be added to the coordination problem with different objective functions and constraints. The development in battery storage technology is promising for the utility scale. In future studies, battery storage could be considered to mitigate risks from the shortterm operational and long-term investment perspectives.

Acknowledgement This paper presents the scientific results of the project "Intelligent system for trading on wholesale electricity market" (SMARTRADE), co-financed by the European Regional Development Fund (ERDF), through the Competitiveness Operational Programme (COP) 2014-2020, priority axis 1 - Research, technological development and innovation (RD\&I) to support economic competitiveness and business development, Action 1.1.4 - Attracting high-level personnel from abroad in order to enhance the RD capacity, contract ID P_37_418, no. 62/05.09.2016, beneficiary The Bucharest University of Economic Studies.

Open Access This article is distributed under the terms of the Creative Commons Attribution 4.0 International License (http:// creativecommons.org/licenses/by/4.0/), which permits unrestricted use, distribution, and reproduction in any medium, provided you give appropriate credit to the original author(s) and the source, provide a link to the Creative Commons license, and indicate if changes were made.

\section{References}

[1] Botterud A, Wang J, Bessa RJ et al (2010) Risk management and optimal bidding for a wind power producer. In: Proceedings of IEEE 2010 power and energy society general meeting, Minneapolis, USA, 25-29 July 2010, pp 1-8

[2] Zugno M, Morales JM, Pinson P et al (2013) Pool strategy of a price-maker wind power producer. IEEE Trans Power Syst 28(3):3440-3450

[3] Morales JM, Conejo AJ, Pérez-Rui J (2010) Short-term trading for a wind power producer. IEEE Trans Power Syst 25(1):554-564

[4] Li T, Shahidehpour M, Li Z (2007) Risk-constrained bidding strategy with stochastic unit commitment. IEEE Trans Power Syst 22(1):449-458

[5] Al-Awami AT, El-Sharkawi MA (2011) Coordinated trading of wind and thermal energy. IEEE Trans Sustain Energy 2(3):277-287

[6] Balancing T, Center S (2014) Day-ahead market. https://www. pmum.gov.tr/pmumportal/xhtml/gop.xhtml. Accessed 24 August 2014

[7] Lew D, Milligan M, Jordan G et al (2011) The value of wind power forecasting. In: Proceedings of 91st American meteorological society annual meeting, Washington DC, USA, 26 January 2011, 13pp

[8] Khodayar ME, Shahidehpour M (2013) Stochastic price-based coordination of intrahour wind energy and storage in a generation company. IEEE Trans Sustain Energy 4(3):554-562

[9] Nieta AA, Contreras J, Munõz JI (2013) Optimal coordinated wind-hydro bidding strategies in day-ahead markets. IEEE Trans Power Syst 28(2):798-809

[10] García-González J, Muela RMR, Santos LM et al (2008) Stochastic joint optimization of wind generation and pumped- storage units in an electricity market. IEEE Trans Power Syst 23(2):460-468

[11] Wang Q, Wang J, Guan Y (2013) Price-based unit commitment with wind power utilization constraints. IEEE Trans Power Syst 28(3):2718-2726

[12] Pappala VS, Erlich I, Rohrig K (2009) A stochastic model for the optimal operation of a wind-thermal power system. IEEE Trans Power Syst 24(2):940-950

[13] Xie J, Fu R, Du P et al (2011) Wind-thermal unit commitment with mixed-integer linear EENS and EEES formulations. In: Proceedings of 4th international conference on electric utility deregulation and restructuring and power technologies (DRPT), Shandong, China, 6-9 July 2011, pp 1808-1815

[14] Pineda S, Conejo AJ (2010) Scenario reduction for risk-averse electricity trading. IET Gener Transm Distrib 4(6):694-705

[15] Alabdulwahab A, Abusorrah A, Zhang X et al (2017) Stochastic security-constrained scheduling of coordinated electricity and natural gas infrastructures. IEEE Syst J 11(3):1674-1683

[16] Xu X, Yan Z, Shahidehpour M et al (2018) Power system voltage stability evaluation considering renewable energy with correlated variabilities. IEEE Trans Power Syst 33(3):3236-3245

[17] Wang X, Li Z, Shahidehpour M et al (2017) Robust line hardening strategies for improving the resilience of distribution systems with variable renewable resources. IEEE Trans Sustain Energy. https://doi.org/10.1109/TSTE.2017.2788041

[18] Plazas MA, Conejo AJ (2005) Multimarket optimal bidding for a power producer. IEEE Trans Power Syst 20(4):2041-2050

[19] Ozguner E (2012) Short term electricity price forecasting in Turkish electricity market. Dissertation, Middle East Technical University

[20] Wood AJ, Wollenberg BF (1996) Power generation, operation, and control. Wiley, New York

[21] Wang J, Shahidehpour M, Li Z (2008) Security-constrained unit commitment with volatile wind power generation. IEEE Trans Power Syst 23(3):1319-1327

[22] Ortega-Vazquez M, Kirschen D (2009) Estimating spinning reserve requirements in systems with significant wind power generation penetration. IEEE Trans Power Syst 24(1):114-124

[23] Bazmohammadi S, Foroud AA, Bazmohammadi N (2012) Stochastic-based risk-constrained optimal self-scheduling for a generation company in electricity market. In: Proceedings of 20th Iranian conference on electrical engineering (ICEE2012), Tehran, Iran, 15-17 May 2012, pp 467-472

Aycan AYDOĞDU received his B.S. and M.S. degrees from Middle East Technical University (METU), Turkey, in 2011 and 2014, respectively. He is currently a senior systems engineer in energy sector at ASELSAN A.Ş, Ankara, Turkey. His research interests are real-time monitoring and market operation of power systems.

Osman Bülent TÖR received his B.S., M.S., and Ph.D. degrees from Middle East Technical University (METU), Turkey, in 1998, 2001 and 2008, respectively. $\mathrm{He}$ is working as managing partner and director at EPRA Electric Energy Co., Ankara, Turkey. His research interests include power system analysis, market operations of power systems, and optimization techniques.

Ali Nezih GÜVEN received the B.S. degree from Middle East Technical University (METU), Ankara, Turkey, in 1979 and the M.S. and Ph.D. degrees from the Ohio State University, Columbus, USA in 1981 and 1984, respectively. He is currently a professor in the Department of Electrical and Electronics Engineering at METU, Ankara, Turkey. His research interests are the analysis, design, and operation of power systems, and distribution automation. 